\title{
The Application Value of Optical Coherence Tomography in Primary Acute Angle Closure Glaucoma
}

\author{
Li Gao* and Xiaodong Tang \\ Department of Ophthalmology, Central Hospital of Liaohe Oil Field, Panjin, Liaoning 124010, China
}

\begin{abstract}
Objective: To investigate the diagnostic value of optical coherence tomography (OCT) in the diagnosis of acute primary angle-closure glaucoma (APACG). Method: 52 eyes were chosen for OCT applications at different stages of APACG and compared with the normal control group. The peripapillary retinal nerve fiber layer (RNFL) between the two groups was compared. Results: The average thickness of RNFL APACG was significantly thicker during the earlier phase of treatment and thinner after 6 months of treatment as compared to the normal control group. Conclusion: OCT can reflect the change in the thickness of glaucoma RNFL, and can provide more information for clinical early diagnosis of glaucoma and is a worthy application of the inspection method.
\end{abstract}

\author{
KEYWORDS \\ Glaucoma \\ Optical coherence tomography \\ Retinal nerve fiber layer
}

\section{Introduction}

In this study, retinal nerve fiber layer (RNFL) was performed in 52 patients (52 eyes) with acute primary angleclosure glaucoma (APACG) and compared with the normal control group. The changes in RNFL thickness were observed.

\section{Materials and methods 2.1. Research object:}

52 patients (52 eyes) in our hospital from July 2013 to July 2015 were chosen. Patients (eyes) treated with monocular for the first time, included 20 males and 32 females, with average age of $60.8+9.2$ years. The inclusion criteria included all patients with typical first monocular APACG symptoms having signs including redness, eye pain, decreased visual acuity, real angle closure, moderate pupil expansion of fixed, intraocular pressure (IOP) $>21 \mathrm{mmHg}$ with a first attack within less than 6 hours, within 24 hours

Copyright $\odot 2016$ Li Gao and Xiaodong Tang

doi: $10.18686 /$ aem.v5i4.14

Received: September 10, 2016; Accepted: November 19, 2016; Published online: December 30, 2016

This is an open-access article distributed under the terms of the Creative Commons Attribution Unported License (http://creativecommons.org/ licenses/by-nc/4.0/), which permits unrestricted use, distribution, and reproduction in any medium, provided the original work is properly cited.

${ }^{\star}$ Corresponding author: Department of Ophthalmology, Central Hospital of Liaohe Oil Field, Panjin, Liaoning 124010, China. E-mail: gaoli2016@yeah.net after drug treatment after remission, and having normal field of vision excluding those that can cause retinal thinning disease, such as high myopia. 52 cases (52 eyes), 23 male and 29 females aged from 59.78 to 41 years old, with an average age of 29 years old were chosen for the normal control group. Subjects were chosen in accordance with the following criteria: (1) Uncorrected visual acuity greater than or equal to 1.0 or best corrected visual acuity of 1.0 and refractive error is less than or equal to 3D; (2) Normal intraocular pressure (IOP) and no family history of glaucoma; (3) Ocular examination was normal, excluding retinal and optic nerve diseases; (4) Without any history of eye surgery; and (5) Not suffering from diabetes or having a history of hypertension in the family. Comparing between the 2 groups, the age difference was not statistically significant $(p>0.05)$. All of the subjects were informed and consented to this research [1].

\subsubsection{Test duration}

Pressure changes on the day after the treatment of corneal transparency and intraocular treatments, after 1 week, 1 month, 3 months, and 6 months ( 5 times) were observed.

\subsection{Method}

\subsubsection{Drug treatment}

Eye drops with $0.5 \%$ nitrate pilocarpine were applied according to ocular and systemic conditions of the patients (Bausch \& Lomb Freda Pharmaceutical Co., Ltd., Shandong, batch number: 07041401), 1\% brinzolamide eye 
drops (Alcon, Belgium, batch number: 07G29I), 2\% hydrochloric acid Timolol eye drops (Otsuka Pharmaceutical Co., Ltd., China, batch number: 8J83E), 20\% mannitol injection (Baite Medical Co., Ltd., Shanghai, batch number: S0701057), methazolamide tablets (Hangzhou Bay Healthcare Ling Pharmaceutical Co., Ltd., batch number: 070403) and other drug treatments were applied. Intraocular pressure (IOP) application was stabilized according to the real angle adhesion for patients undergoing surgical treatment. Real angle adhesion was greater than or equal to $1 / 2$ circle (25 eyes), complex trabeculectomy and chamber angle synechiae less than $1 / 2$ circumference ( 1 eyes) of peripheral iris resection [2].

\subsubsection{Research method}

Patients underwent routine examinations with the Department of Ophthalmology, such as slit lamp examination, refraction and ocular axis measurement, and Goldmann intraocular pressure measurements. Patients were treated by the same glaucoma specialist. The angle of the OCT was detected using the Scheie method. RNFL thickness was detected by using optical coherence tomography (OCT3000) and Stratus (CarlZeiss, Germany). Using the scanning program (RNFL Thickness 3.4) to scan the entire research object, results recorded clear and stable images for measurement. The average RNFL thickness and the average thickness of the whole week were obtained by using 3.46 $\mathrm{mm}$ as the center of the optic papilla (Table 1).

\subsection{Statistical analysis}

SPSS 13 software was used for data analysis, $t$ test evaluation and analysis of variance. Results $(\bar{x}+s)$ indicated that the difference was statistically significant for $p<0.05$.

\section{Results}

OCT images of APACG in any stage of the disease and RNFL images showed that the upper and lower sides of the temporal and nasal sides were thin. RNFL measurements in APACG patients admitted to the hospital after normal intraocular pressure $(0-6 \mathrm{~h})$, and after 1 week were compared. The results were statistically significant $(p<$ 0.05 ). With the development of the disease, RNFL is obviously thicker. After 1 and 6 months, RNFL thickness in the APACG patients were compared with the normal control group and the results were not statistically significant $(p>$
0.05). RNFL thickness seemed to be stable. Patients with APACG were reviewed 6 months after normal intraocular pressure for RNFL thickness comparison and it was statistically significant. However, the other three observations were not statistically significant.

In the APACG group, RNFL thickness increased with acute attacks. After IOP fell to the normal range after 1 week, RNFL thickness gradually decreased. Normal intraocular pressure stabilized after 3 months and 6 months as long as RNFL thickness stabilized and with statistically significant difference as compared to the normal control group [3].

\section{Discussion}

Glaucoma is an elevated IOP caused by optic papilla damage and visual field defect disease. Clinical research confirmed that retinal nerve fiber layer (RNFL) and thinning was earlier than physiological depression expansion and visual field defect detection. Early diagnosis of glaucoma in the past used morphological examination methods, mainly by measuring local or diffused RNFL defects. This would take time before mydriasis and is limited to many conditions, such as lighting conditions and experience of the observer. At present, the application of RNFL for OCT thickness detection can provide objective data and make up for the shortcomings of the previous detection methods which have been reported in China and abroad. Studies have confirmed that RNFL damage in early glaucoma optic disc could cause visual field changes earlier and the use of OCT RNFL can then visualize optic nerve damage. This study used OCT measurements of RNFL in patients with early RNFL thickness through clinical and experimental research with APACG. Peripapillary retinal nerve fiber layer thickness of patients admitted to the hospital after the treatment of corneal transparency by OCT was thicker than that of normal controls in the analysis of acute elevated IOP caused by edema of the nerve fiber layer. Optical disc edema may be due to elevated IOP in the cribriform plate anterior area of vascular compression, resulting in severe ischemia and hypoxia caused by the edema. Within a short time, acute IOP elevation can be caused by shaft cable transport fast block, microsomes and dense bodies in axon savings resulting in optical disc edema and a change in RNFL thickness. After controlling IOP, the cause of retinal edema was eliminated and RNFL thickness

Table 1. Comparison of RNFL thickness in different stages of APACG patients.

\begin{tabular}{cccccc}
\hline & Temporal & Upper & Nasal side & Below & Mean \\
\hline At the time of admission & $84.37 \pm 13.63$ & $138.64 \pm 14.23$ & $72.98 \pm 16.02$ & $127.11 \pm 17.63$ & $105.21 \pm 10.73$ \\
After normal IOP $(0-6 \mathrm{~h})$ & $93.54 \pm 19.13$ & $160.23 \pm 16.83$ & $85.02 \pm 12.62$ & $143.95 \pm 19.03$ & $126.84 \pm 17.43$ \\
1 week after normal IOP & $91.33 \pm 10.92$ & $158.29 \pm 19.04$ & $81.87 \pm 14.08$ & $139.04 \pm 13.28$ & $113.63 \pm 16.65$ \\
1 month after normal IOP & $81.13 \pm 15.54$ & $135.99 \pm 19.32$ & $70.09 \pm 15.33$ & $122.88 \pm 12.88$ & $104.26 \pm 12.78$ \\
6 months after normal IOP & $80.34 \pm 16.78$ & $135.03 \pm 13.72$ & $67,82 \pm 14.98$ & $120.78 \pm 14.52$ & $102.53 \pm 16.46$ \\
\hline
\end{tabular}

*Acute onset less than 6 hours measured data 
of APACG patients gradually decreased and tended to be stable 1 week after reaching normal IOP. APACG patients' RNFL thickness was thicker than that of the normal group. 1 month after IOP treatment, average retinal thickness of the patients were slightly thicker than that of the normal control group, with no significant difference. After 3 and 6 months, the average retinal thicknesses of the patients were slightly thinner than that of the normal control group. In our observations, we found that high IOP applied within a short time will not cause the RNFL to be significantly thinner.

We concluded that the possible cause of acute elevated IOP causes RNFL edema. In addition, IOP within a short time can cause shaft carrying cable fast block, accumulation of microsomes and dense bodies in axons, resulting in optical disc edema, and increase in the RNFL thickness. OCT images of patients with early RNFL APACG showed optic and peripheral retinal hyperemia, edema, and increased thickness of RNFL. According to the results, considering the possibility that the IOP dropped to normal, retinal edema gradually recovered, and stabilized after a month. Patients admitted with a diagnosis of pre-retinal edema caused by retinal thickness increase had their IOPs returning to normal after 6 months of retinal edema. In addition, the consideration was not because APACG-induced retinal damage is a long-term and lasting image manifestation. All of these require us to carry out the relevant experimental study in order to find out the real cause.

Early diagnosis of glaucoma has been the problem of ophthalmic OCT due to the detection of RNFL thickness and it can directly provide objective test data, which has the advantage of non-contact, non-damaging, high resolution means of inspection with widespread clinical acceptance [4]. However, clinical diagnosis of glaucoma must be according to the comprehensive analysis of IOP and visual field, and if necessary, further examination would have to be done to improve the diagnosis. It is worth noting that the application value of OCT in APACG is certain and it is suitable to be promoted and applied.

\section{Conflicts of interest}

These authors have no conflicts of interest to declare.

\section{Authors' contributions}

These authors contributed equally to this work.

\section{References}

1. Ge J. Pediatric ophthalmology practice efficiency. Ophthalmology. People's Health Publishing House; 2006:248.

2. Ge J, Luo RJ, Liu X. Preliminary study on the thickness of retinal nerve layer thickness of the retinal nerve layer of optical coherence tomography. Clinical Ophthalmology. 2013;17(6):331-335.

3. Liu X, Huang SZ, Ling YL. Patient-reported outcome measures in paediatric ophthalmology. Journal of Modern Ophthalmology. 2011;6:481-483.

4. Liu X, Li M, Huang JJ. Research on the thickness of retinal nerve fiber layer in patients with acute angle closure glaucoma. Chinese Journal of Practical Ophthalmology. 2014;26(6):562-565. 